\title{
Characterization and Secretory Expression of a Thermostable Tannase from Aureobasidium melanogenum T9: Potential Candidate for Food and Agricultural Industries
}

\section{OPEN ACCESS}

Edited by: Shangyong Li,

Qingdao University, China

Reviewed by: Yasser Gaber,

Beni-Suef University, Egypt Satya P. Singh,

Saurashtra University, India

*Correspondence:

Wei-Qing Song

songweiqing68@163.com

Hai-Xiang Zhou

pro.zhouhaixiang@163.com

Specialty section:

This article was submitted to

Bioprocess Engineering,

a section of the journal

Frontiers in Bioengineering and

Biotechnology

Received: 02 September 2021 Accepted: 30 December 2021

Published: 08 February 2022

Citation:

Liu L, Guo J, Zhou X-F, Li Z, Zhou H-X and Song $W$ - $Q$ (2022) Characterization and Secretory Expression of a

Thermostable Tannase from Aureobasidium melanogenum T9: Potential Candidate for Food and Agricultural Industries.

Front. Bioeng. Biotechnol. 9:769816.

doi: 10.3389/fbioe.2021.769816

\author{
Lu Liu ${ }^{1,2}$, Jing Guo ${ }^{1}$, Xue-Feng Zhou ${ }^{3}$, Ze Li ${ }^{4}$, Hai-Xiang Zhou ${ }^{1 *}$ and Wei-Qing Song ${ }^{1 *}$ \\ ${ }^{1}$ Department of Clinical Laboratory, Qingdao Municipal Hospital, Qingdao, China, ${ }^{2}$ School of Medicine and Pharmacy, Ocean \\ University of China, Qingdao, China, ${ }^{3}$ Clinical Trial Research Center, The Affiliated Central Hospital of Qingdao University, \\ Qingdao, China, ${ }^{4}$ College of Advanced Agricultural Sciences, Linyi Vocational University of Science and Technology, Linyi, China
}

Being a key industrial enzyme, tannase is extensively applied in various fields. Despite the characterizations of a large number of tannases, there are hardly a few tannases with exceptional thermostability. In this detailed study, a tannase-encoding gene named $\tan A$ was identified from Aureobasidium melanogenum T9 and heterologously expressed in Yarrowia lipolytica host of food grade. The purified tannase TanA with a molecular weight of above $63.0 \mathrm{kDa}$ displayed a specific activity of $941.4 \mathrm{U} / \mathrm{mg}$. Moreover, TanA showed optimum activity at $60^{\circ} \mathrm{C}$ and $\mathrm{pH} 6.0$. Interestingly, TanA exhibited up to $61.3 \%$ activity after incubation for $12 \mathrm{~h}$ at $55^{\circ} \mathrm{C}$, signifying its thermophilic property and distinguished thermostability. Additionally, TanA was a multifunctional tannase with high specific activities to catalyze the degradation of various gallic acid esters. Therefore, this study presents a novel tannase, TanA, with remarkable properties, posing as a potential candidate for food and agricultural processing.

Keywords: tannin, tannase, thermostability, Aureobasidium melanogenum, Yarrowia lipolytica

\section{INTRODUCTION}

As a type of polyphenol compound generated to resist a bad growth environment, tannins are extensively present in higher plants (Chung et al., 1998). The formation of stable complexes with various biological macromolecules, such as polysaccharides and proteins, provides lower nutritive value to food, thus making them nutritionally undesirable (Pan et al., 2020). According to some reports, tannins are one of the primary causes of low food intake, slow growth, low level of fodder utilization rate, and low protein breakdown in laboratory animals (Becker and Makkar, 1999; Min et al., 2005; Sengil and Oezacar, 2009). Therefore, the removal of tannins becomes an integral process during food and feed processing. Fortunately, there still exist some special microorganisms which can grow and reproduce stably in an environment containing tannins, making the full utmost of the secreted enzymes to decompose tannins into small-molecule phenolic compounds (Chhokar et al., 2013).

Tannase (EC 3.1.1.20), also known as tannin acylhydrolase, is a hydrolase that can effectively hydrolyze ester bonds and carboxyphenol bonds in hydrolyzable tannins, including ellagitannins (ETs), gallotannins (GTs), and other gallic acid esters to produce polyphenol compounds such as gallic acid (Aguilar and Gutierrez-Sanchez, 2001; Zhang et al., 2015; Govindarajan et al., 2016). The 
enzymatic degradation of tannins is considered a safe and environmentally friendly method that can effectively overcome the shortcomings of traditional hydrolysis methods and significantly improve the yield and purity of products (Raghuwanshi et al., 2011; Jana et al., 2013; Ghosh and Mandal, 2015; Ebrahimzade et al., 2018). Therefore, tannases have played a significant role in industrial production, especially in the fields of feed, food, brewing, and pharmaceuticals (Chhokar et al., 2013; Wang et al., 2022). Rapeseed meal is considered to be one of the sources of high-quality plant protein feed due to its high protein content and balanced amino acid composition. However, rapeseed meal contains a variety of anti-nutritional factors, like tannins, that seriously affect its palatability as well as nutritive value, severely limiting the application of rapeseed meal in animal feeding (Shim et al., 2003). Interestingly, Lorusso et al. (1996) reported that the tannase produced by Trametes versicolor could degrade more than $80 \%$ of tannins in rapeseed meal within $30 \mathrm{~min}$, strengthening the application of tannase in the processing of rapeseed meal feed. Furthermore, the transformation of ester catechins (also belonging to tannins) in green tea to non-ester catechins for improving the taste of tea drinks is another important use for tannases (Ozturk et al., 2016). Therefore, these properties of tannases are of great interest among researchers.

Currently, owing to the characteristics of biochemical diversity and liable genetic manipulations, microbial fermentation is the major approach for the continuous production of tannases (Beniwal et al., 2013). Numerous studies have been conducted for screening tannase-producing microorganisms, of which bacteria and fungi have shown enhanced enzyme production ability (Banerjee et al., 2012; Kanpiengjai et al., 2019). However, tannases produced by bacteria demonstrated limitations in low activity and poor thermostability, imposing major concerns of large-scale application (Rivas et al., 2019). Thus, there are relatively scarce studies of bacterial tannases. On the other hand, the complex heredity of fungi makes genetic manipulations difficult (Beniwal et al., 2010). These collectively present challenges in identifying tannases with better properties for industrial application-for example, tannases play a pivotal role in green tea deep processing to improve the appearance, aroma, and flavor of tea and the extraction efficiency of polyphenols (Cao et al., 2019). During the extraction of green tea, the extraction yield increased with increasing temperature within a certain range (Shao et al., 2020). However, since the enzyme catalysis reaction is usually temperature-limited, tea processing and extraction consume a prolonged time (over $2 \mathrm{~h}$ ) at a lower range of temperature from 30 to $40^{\circ} \mathrm{C}$, thereby reducing product quality and production efficiency (Hong et al., 2013). Therefore, it becomes necessary to screen genetically stable microorganisms with the capability to produce tannases of high vigor and thermal stability. Jana et al. (2013) reported that the high-temperature and solvent-resistant tannase produced from Bacillus subtilis PAB2 had a half-life of up to $4.5 \mathrm{~h}$ in an environment of $60^{\circ} \mathrm{C}$. Shao et al. (2020) screened Aspergillus niger FJ0118 that could generate tannase rAntan 1 with strong temperature tolerance, and its half-life at $60^{\circ} \mathrm{C}$ was persisting for about $5.4 \mathrm{~h}$. Nevertheless, the majority of the reported thermostable tannases cannot reach food grade due to safety concerns.

Aureobasidium melanogenum T9, with the capability to degrade tannins, has been successfully isolated from the starter of red wine (Zhang et al., 2019). In this study, the gene of encoding tannin-degrading enzyme, $\tan A$, was identified and heterologously expressed in the Yarrowia lipolytica yeast of food grade. The detailed characterization of tannase TanA displayed superior thermal stability and unique robustness, thus laying a solid foundation for its application in the industry.

\section{MATERIALS AND METHODS}

\section{Bioinformatics Analysis of TanA}

In the previous research, the genome of A. melanogenum T9 strain was sequenced and annotated by Novogene (Zhang et al., 2019); in silico studies were performed to determine the encoding gene sequence of TanA. By using HMMER3, the protein sequence was compared with carbohydrate-active enzyme database to acquire the message. The filter condition was set to $E$ value $<1 \mathrm{E}^{-5}$. The theoretical molecular weight $(\mathrm{Mw})$ and isoelectric point ( $\mathrm{pI}$ ) value were obtained through online prediction (http:// web.expasy.org/compute_pi/). SignalP 4.1 server was applied for signal peptide analysis (http://www.cbs.dtu.dk/services/SignalP$4.1 /$ ). NetNGlyc 1.0 server was applied to predict N-glycosylation sites (http://www.cbs.dtu.dk/services/NetNGlyc/). The conserved domain database of the National Center for Biotechnology Information (NCBI, Bethesda, MD, United States) was used for domain analysis (https://www.ncbi.nlm.nih.gov/cdd).

To study the evolutionary relationship among TanA and other tannases derived from microorganisms, a bootstrapped phylogenetic tree was constructed by neighbor-joining method with MEGA 6.0 software (Tamura et al., 2013) on the basis of the amino acid sequences of related tannases obtained from NCBI (https://www.ncbi.nlm.nih.gov/). Multiple sequence alignment was carried out using DNAMAN 6.0 software (Lynnon Biosoft, Foster City, CA, United States). The 3D model of tannase TanA (accession no.: QEP28943.1) of A. melanogenum T9 was built on the basis of the crystal structure of feruloyl esterase AoFaeB from Aspergillus oryzae RIB40 (PDB: 3WMT) (Suzuki et al., 2014), using basic modeling module in modeller 9. 20. Fifty homologous models were firstly constructed, and one model with the highest score was obtained.

\section{Secretory Expression of TanA}

The $\tan A$ gene without signal sequence was synthesized by Synbio Technologies (Suzhou, China) following codon optimization. The linearized DNA fragment was successfully transformed into the $Y$. lipolytica URA $^{-}$strain (Zhang et al., 2018). The Y. lipolytica cultivation and the purification steps of TanA generally followed those of Zhou et al. (2020). After culturing in GPPB liquid media $[30.0 \mathrm{~g} / \mathrm{L}$ glucose, $2.0 \mathrm{~g} / \mathrm{L}$ yeast extract, $1.0 \mathrm{~g} / \mathrm{L}$ $\left(\mathrm{NH}_{4}\right)_{2} \mathrm{SO}_{4}, \quad 3.0 \mathrm{~g} / \mathrm{L} \quad \mathrm{K}_{2} \mathrm{HPO}_{4}, \quad 2.0 \mathrm{~g} / \mathrm{L} \quad \mathrm{KH}_{2} \mathrm{PO}_{4}, \quad 0.1 \mathrm{~g} / \mathrm{L}$ $\mathrm{MgSO}_{4} \cdot 7 \mathrm{H}_{2} \mathrm{O}, \mathrm{pH}$ 6.8] for $72 \mathrm{~h}$ at $30^{\circ} \mathrm{C}$, the tannase activities of the positive transformants were discovered (Pan et al., 2020). In comparison, the recombinant strain 72 displayed optimal 
extracellular activity. Afterwards, $100 \mathrm{ml}$ of the supernatant was concentrated firstly by ultrafiltration with a centrifugal filter $3 \mathrm{~K}$ device (Millipore, Burlington, MA, United States), and then it was injected into the His60 Ni Superflow affinity chromatography column purchased from Clontech Laboratories, Inc. (TaKaRa, Dalian, China), which was already equilibrated with $50 \mathrm{mM}$ phosphate buffer ( $\mathrm{pH} 7.4$ ) containing $300 \mathrm{mM} \mathrm{NaCl}$ and $20 \mathrm{mM}$ imidazole. After successive washing using the same buffer containing $40 \mathrm{mM}$ imidazole and elution using the same buffer containing a linear gradient of imidazole (50-400 mM), the $6 \times$ His-tagged TanA was obtained (Zhang et al,, 2021). The fractions with tannase activity were pooled and concentrated via Millipore centrifugal filter $3 \mathrm{~K}$ device. Meanwhile, the enzyme solution was desalted, and the buffer was replaced with $50 \mathrm{mM}$ Tris- $\mathrm{HCl}$ ( $\mathrm{pH}$ 7.4) for further characterization of TanA. During the purification of TanA, a BCA protein assay kit (TaKaRa, Dalian, China) was used to determine the total protein concentration. Moreover, $12 \%$ sodium dodecyl sulfate-polyacrylamide gel electrophoresis (SDS-PAGE) was used to verify the Mw and purity of TanA.

\section{Determination of Tannase Activity}

TanA activity was determined by measuring the amount of gallic acid produced in the reaction, generally according to the description in Sharma et al. (2000). Briefly, $0.5 \mathrm{ml}$ of properly diluted enzyme solution was mixed with $4.5 \mathrm{ml}$ of $0.5 \%(\mathrm{w} / \mathrm{v})$ propyl gallate in $100 \mathrm{mM} \mathrm{Na}_{2} \mathrm{HPO}_{4}$-citric acid buffer ( $\mathrm{pH} 5.0$ ) at $40^{\circ} \mathrm{C}$ for $10 \mathrm{~min}$. The gallic acid content was measured by the formation of chromogen violet staining between $0.667 \%(\mathrm{w} / \mathrm{v})$ rhodanine in ethanol and gallic acid, followed by recording the absorbance at $520 \mathrm{~nm}$ by a spectrophotometer. One unit of enzyme activity was defined as the amount of tannase that generates $1 \mu \mathrm{mol}$ gallic acid per minute.

\section{Temperature and $\mathrm{pH}$ Properties for TanA Activity}

The optimum temperature was determined by carrying out a TanA-mediated hydrolysis reaction at different temperatures ranging from 20 to $70^{\circ} \mathrm{C}$. For the purpose of assessing thermostability, the residual activity of purified TanA was investigated after incubating at $20-70^{\circ} \mathrm{C}$ for $12 \mathrm{~h}$. Additionally, propyl gallate solutions were prepared with $100 \mathrm{mM}$ disparate $\mathrm{pH}$ buffers (glycine- $\mathrm{NaOH}, \mathrm{pH} 8.5-11.0 ; \mathrm{Na}_{2} \mathrm{HPO}_{4}$-citric acid, $\mathrm{pH}$ 2.0-8.0) to conduct as substrates of enzymatic reaction for the confirmation of optimum $\mathrm{pH}$. The $\mathrm{pH}$ stability of TanA was surveyed through measuring the rest of enzyme activity after incubation at $40^{\circ} \mathrm{C}$ for $12 \mathrm{~h}$ in buffers of different $\mathrm{pH}$.

\section{Effects of Chemicals and Metal lons on TanA Activity}

The catalytic reactions were performed using propyl gallate liquors with 1 and $5 \mathrm{mM}$ of different compounds or metal ions as substrates. The reaction that took place in a propyl gallate solution without any compound/metal ion was considered the control.

\section{Degradation of Gallic Acid Esters by TanA}

The substrate specificity of tannase TanA was analyzed according to its specific activities assayed under the standard method described above using $0.5 \%(\mathrm{w} / \mathrm{v})$ of different gallic acid esters as substrates, including methyl gallate (MG), propyl gallate (PG), tannic acid (TA), gallocatechin gallate (GCG), epicatechin gallate (ECG), and epigallocatechin gallate (EGCG). The chemical structures of the substrates are displayed in Supplementary Figure S1. Meanwhile, the Michaelis constants $\left(K_{\mathrm{m}}\right)$ were calculated against these substrates based on the doublereciprocal plot of Lineweaver-Burk (Lineweaver and Burk, 1934).

In order to further investigate the capability of TanA to degrade these substrates, excess tannase TanA ( $0.5 \mathrm{U}$ per milligram of each substrate, namely, $2.5 \mathrm{U} / \mathrm{ml}$ of each reaction solution) was incubated with $0.5 \%(\mathrm{w} / \mathrm{v})$ of each gallic acid ester ( $\mathrm{pH} 6.0)$; the catalytic reaction mixtures were stirred continuously at $55^{\circ} \mathrm{C}$ for $2 \mathrm{~h}$. After incubation, the solution of each degraded product was immediately loaded onto the Millipore centrifugal filter $3 \mathrm{~K}$ device to remove the protein and unsolved materials. High-performance liquid chromatography (HPLC) was employed to confirm the transformation of these substrates, and the analysis method was described briefly as follows: Diamonsil $\mathrm{C}_{18}$ column $(3.0 \times 250 \mathrm{~mm}, 5 \mu \mathrm{m})$, wavelength of $278 \mathrm{~nm}$, mobile phase A (acetonitrile) and B (0.5\% acetic acid-water), and flow velocity of $1.0 \mathrm{ml} / \mathrm{min}$ by gradient elution as reported (Ni et al., 2015).

\section{RESULTS}

\section{Bioinformatics Analysis of TanA}

The genomic DNA of $A$. melanogenum T9 strain sequencing illustrated a putative tannase-encoding gene $\tan A$. The deduced amino acid sequence was deposited into the Genebank database, and the accession number QEP28943.1 was provided (Zhang et al., 2019). The open reading frame of $\tan A$ was composed of $1,587 \mathrm{bp}$ encoding a protein of 528 amino acids, of which the first 19 amino acids (highlighted by the blue box in Figure 1) were anticipated as the signal peptide, which was consistent with the secretion feature. The theoretical $\mathrm{pI}$ and $\mathrm{Mw}$ were predicted to be 5.50 and $57.2 \mathrm{kDa}$, respectively. Interestingly, the BLAST tool of NCBI revealed that TanA possessed the conserved domain closely related to the tannase and ferulic acid esterase family (data not shown).

Then, a phylogenetic tree was built to determine the ascription of TanA. As illustrated in Figure 2, the present enzyme TanA, which was on the same branch as the tannase from Pyrenophora triticirepentis (accession no.: PWO06677.1), distinctly pertained to the tannase and feruloyl esterase family. Moreover, sequence comparative analysis was performed among TanA and other members of the tannase and feruloyl esterase family (Figure 1), including the tannase from Trichoderma harzianum (accession no.: KKP04619.1), the tannase from Cadophora sp. DSE1049 (accession no.: PVH72277.1), the tannase from Fusarium langsethiae (accession no.: KPA35627.1), the tannase from $P$. tritici-repentis (accession no.: PWO06677.1), the tannase from Aspergillus ruber CBS 135680 (accession no.: EYE96818.1), the tannase from Rutstroemia sp. NJR-2017a BVV2 (accession no.: PQE10574.1), the tannase from Penicillium camemberti (accession no. 


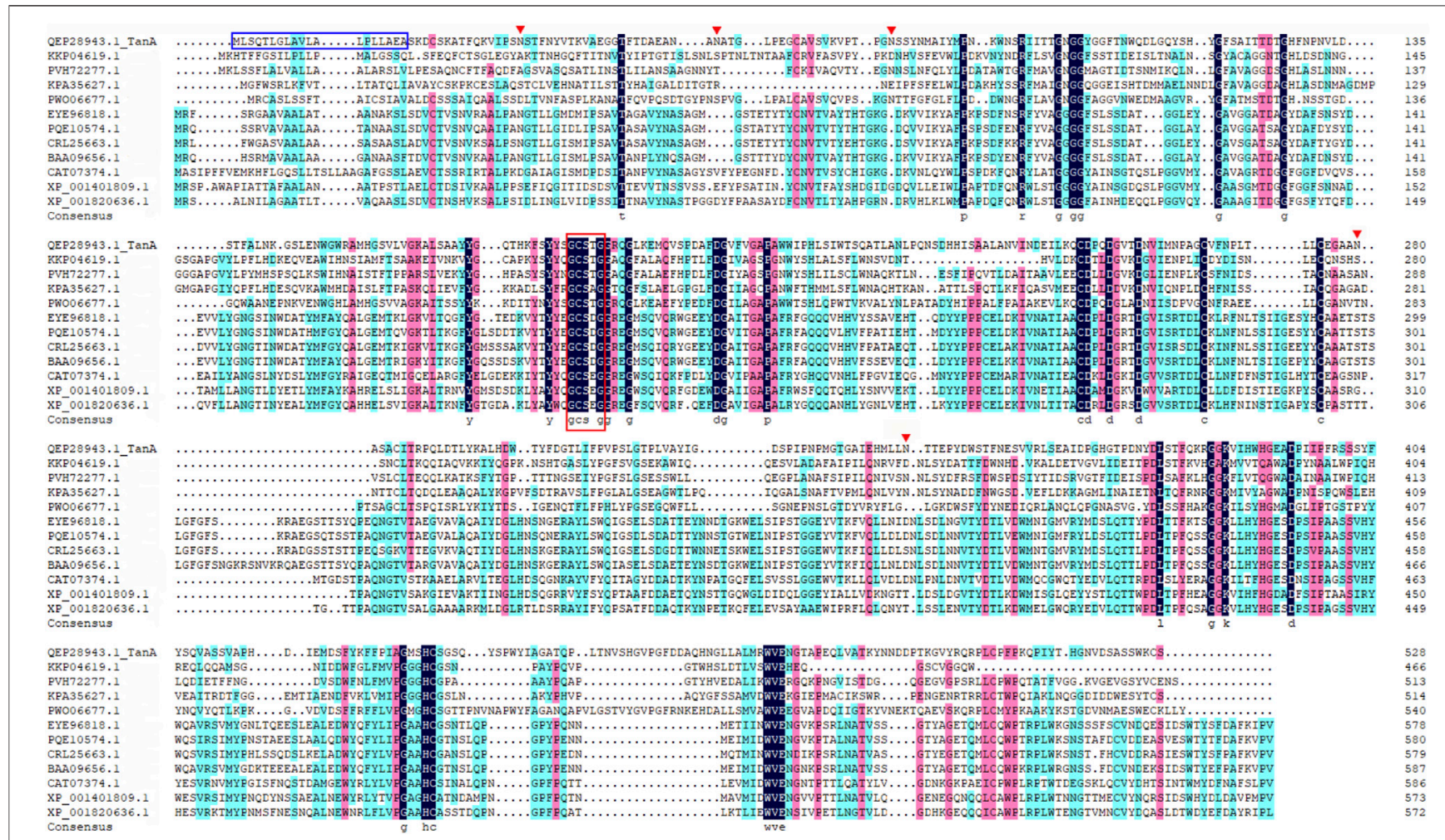

FIGURE 1 | Multiple sequence alignment among TanA and other reported tannases. The signal peptide is boxed in blue, the Gly-X-Ser-X-Gly conserved domain is highlighted by a red box, and the predicted N-glycosylation sites are marked with red inverted triangles.

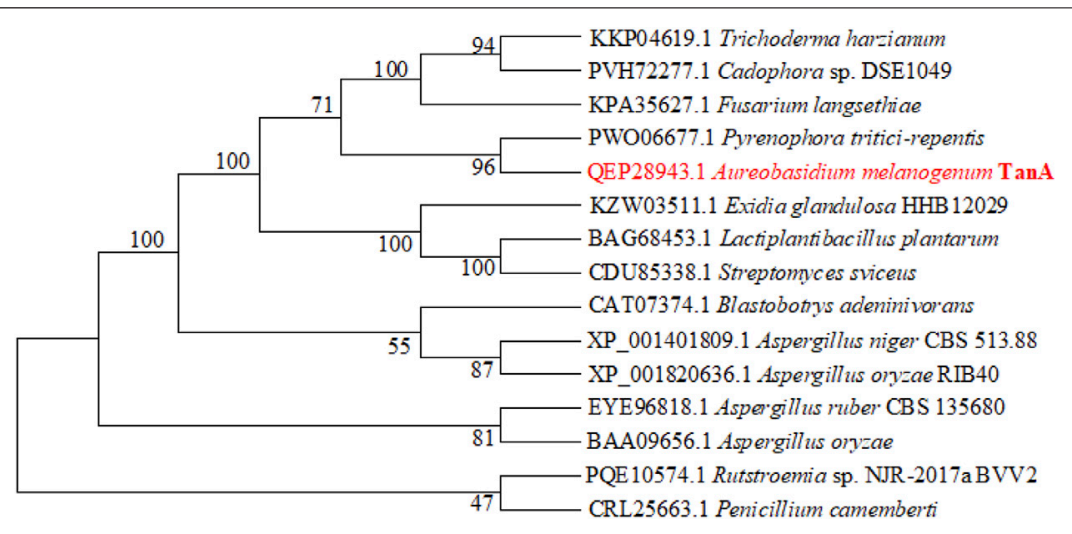

FIGURE 2 | The phylogenetic tree constructed according to the sequences of TanA and other tannases. TanA researched in this study is in red.

CRL25663.1), the tannase from A. oryzae (accession no.: BAA09656.1), the tannase from Blastobotrys adeninivorans (accession no.: CAT07374.1), the tannase from A. niger CBS 513.88 (accession no.: XP_001401809.1), and the tannase from $A$. oryzae RIB40 (accession no.: XP_001820636.1). The multiple sequence alignment showed that TanA retained a typical Gly-XSer-X-Gly conserved domain (represented by the red box in Figure 1). The $3 \mathrm{D}$ structure of TanA in this study has been constructed through homology modeling, and the molecular graphic image was prepared using PyMOL 2.0.3 (Schrödinger, LLC, Portland, OR, United States) and displayed in Supplementary Figure S2.

\section{Secretory Expression of TanA in Y. lipolytica}

The secretory expression of TanA was further studied in the heterogenous host of food grade with significant cellular exocrine ability, Y. lipolytica (Madzak, 2015). The tannase activity was measured as $89.4 \mathrm{U} / \mathrm{ml}$ following $72 \mathrm{~h}$ of incubation in GPPB 
TABLE1 | Purification of tannase TanA.

\begin{tabular}{|c|c|c|c|c|c|}
\hline Purification step & Total protein (mg) & Total activity (U) & Specific activity (U/mg) & Purification fold & Yield (\%) \\
\hline Crude enzyme & 57.5 & $8,940.0$ & 155.5 & 1 & 100 \\
\hline $\mathrm{Ni}$-IDA agarose & 5.81 & $5,469.5$ & 941.4 & 6.05 & 61.2 \\
\hline
\end{tabular}

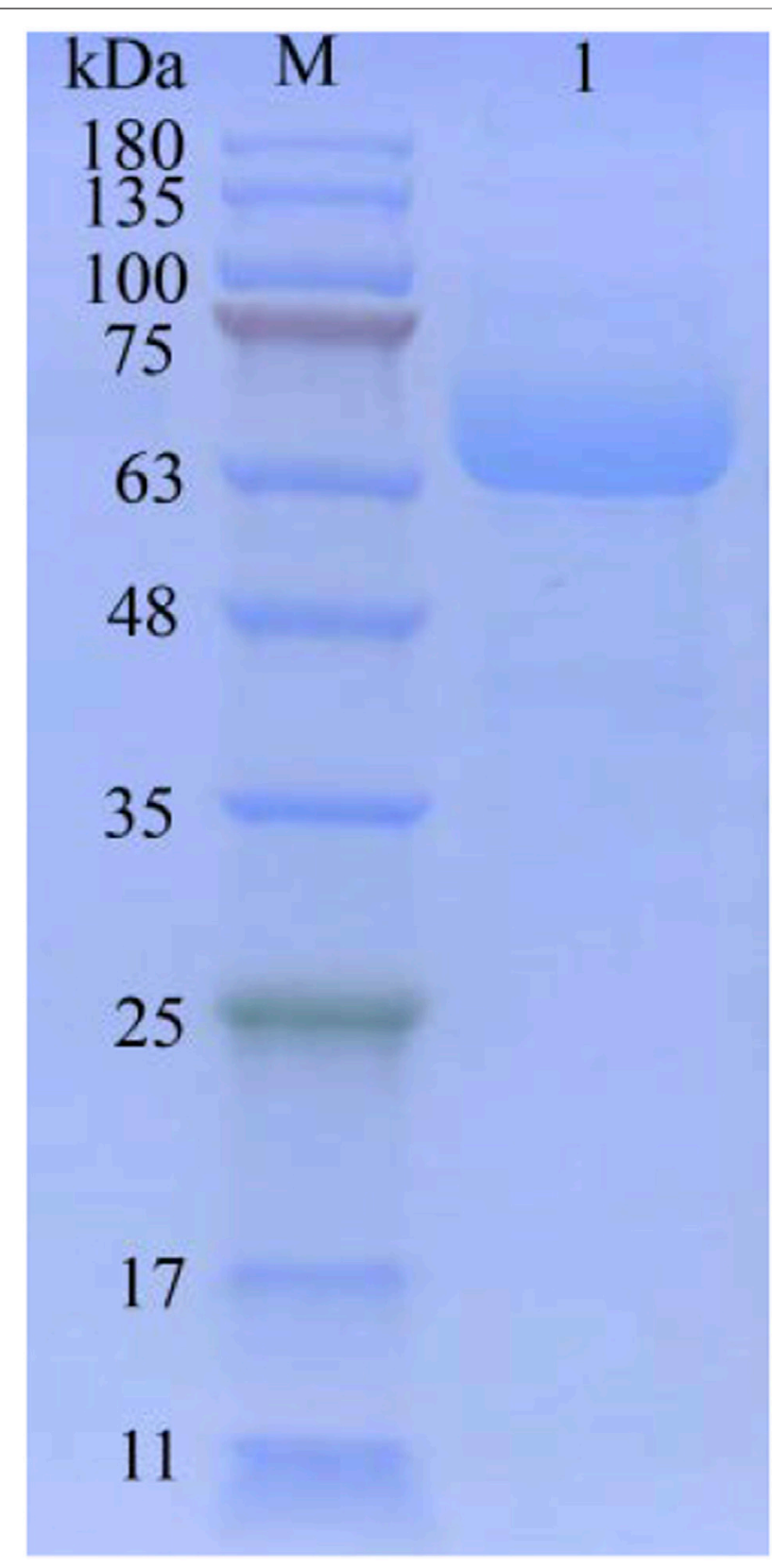

FIGURE 3 I SDS-PAGE of purified TanA. Lane 1: purified TanA; lane M: prestained protein ladder.

medium. The summary of TanA purification is listed in Table $\mathbf{1}$. After Ni-IDA affinity chromatography, the purified TanA was obtained, with a specific activity of $941.4 \mathrm{U} / \mathrm{mg}$. The SDS-PAGE analysis of the purified TanA protein depicted a predominant band observed at approximately $63.0 \mathrm{kDa}$ (Figure 3), a bit larger than the theoretical calculation, which could be possibly due to the glycosylation of the protein. To this end, NetNGlyc 1.0 server was employed to forecast five $\mathrm{N}$-glycosylation recognition sites of TanA (marked with red and inverted triangles in Figure 1). In addition, the fusion of $6 \times$ His-tag introduced several extra amino acids into the recombinant TanA.

\section{Effects of Temperature on TanA Activity}

As demonstrated in Figure 4A, the activity of TanA was linearly correlated to temperature rise, with optimal response at $60^{\circ} \mathrm{C}$ and over $80 \%$ activity retention from 40 to $70^{\circ} \mathrm{C}$. Interestingly, TanA still showed 85.2 and $81.5 \%$ of optimum activity even under the conditions of 65 and $70^{\circ} \mathrm{C}$ (Figure 4A). It was further observed that TanA exhibited impressive stability below $55^{\circ} \mathrm{C}$. It could maintain up to $61.3 \%$ activity even after $12 \mathrm{~h}$ of incubation at $55^{\circ} \mathrm{C}$ (Figure 4B). Therefore, TanA demonstrated thermophilic property with superior thermostability.

\section{Effects of pH on TanA Activity}

As TanA displayed the optimal catalytic activity at $\mathrm{pH} 6.0$ (Figure 5A), the effects of $\mathrm{pH}$ was elucidated. TanA maintained exceeding $70 \%$ of its activity after incubation for $12 \mathrm{~h}$ at $40^{\circ} \mathrm{C}$ within the wide $\mathrm{pH}$ range from 3.5 to 7.5 (Figure 5B), indicating that it possessed favorable stability under acidic to weakly alkaline conditions.

\section{Effects of Chemicals or Metal Ions on TanA Activity}

The effects of various metal ions and chemicals on the activity of TanA are shown in Figure 6. $\mathrm{Cu}^{2+}, \mathrm{Ba}^{2+}, \mathrm{Al}^{3+}$, and $\mathrm{Mg}^{2+}$ strongly inhibited the activity of TanA. However, a slightly activated effect was observed in the presence of $\mathrm{Fe}^{2+}$; the relative activity reached $118.2 \%$ with $5 \mathrm{mM} \mathrm{Fe}{ }^{2+}$. Contrastingly, the enzymatic activity was obviously enhanced by $\mathrm{Zn}^{2+}, \mathrm{Mn}^{2+}$, and $\mathrm{Co}^{2+}$ at both 1 and 5 $\mathrm{mM}$, which was increased to $131.5,145.8$, and $127.5 \%$, respectively, with $5 \mathrm{mM}$ of these cation ions. Other chemical compounds, especially mercaptoethanol (ME), manifested dramatic inactivation effects on TanA, whose activity was reduced to $21.6 \%$ compared to the control when subjected to $1 \mathrm{mM}$ of ME and even was thoroughly lost after $5 \mathrm{mM}$ of ME was added into the reaction solution, which perhaps is attributed to the significant ability of ME to damage disulfide bonds.

\section{Substrate Specificity of TanA and Degradation Product Analysis}

In order to study the substrate specificity of TanA, various gallic acid esters were employed as substrates. The specific activities and 

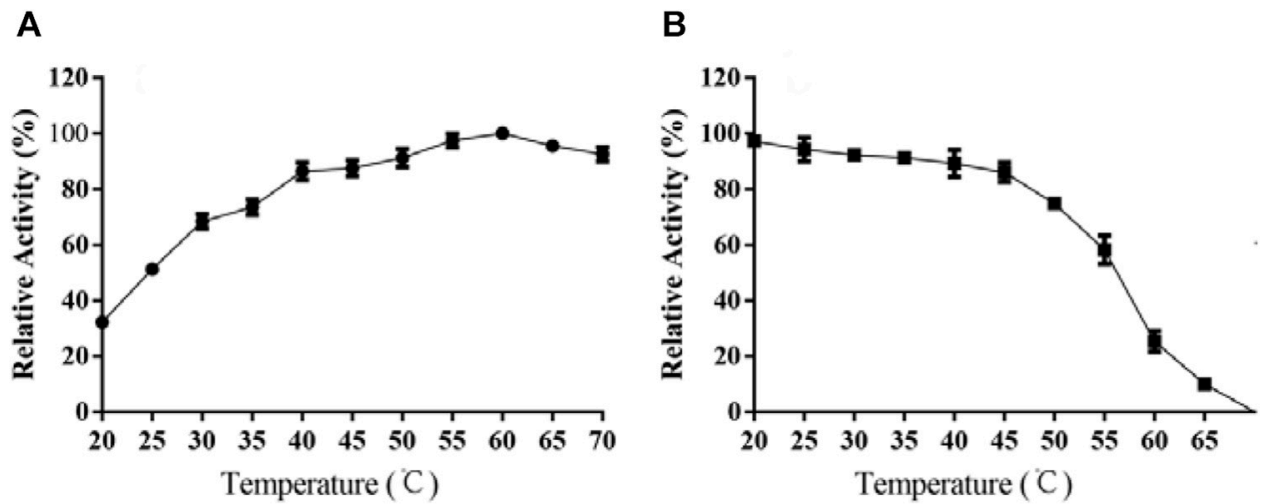

FIGURE 4 | Effects of temperature on the activity (A) and stability (B) of TanA. (A) The optimal temperature of TanA was assessed in the range of $20-70^{\circ} \mathrm{C}$, regarding the activity at optimum temperature as $100 \%$. (B) The temperature stability of TanA was determined by measuring the residual activity after incubation under $20-70^{\circ} \mathrm{C}$ for $12 \mathrm{~h}$; the initial activity was taken as $100 \%$.

A

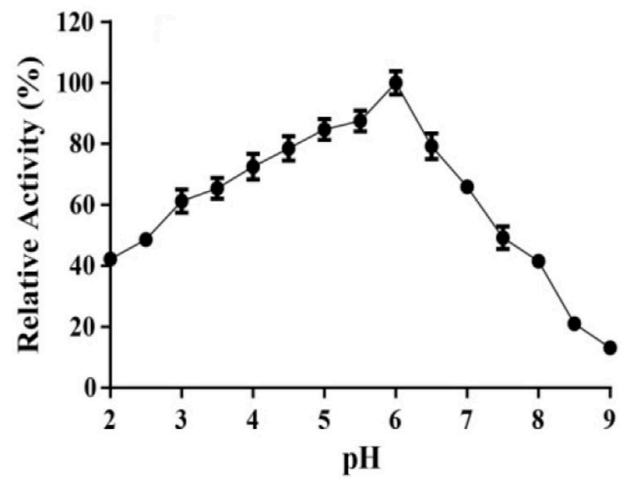

B

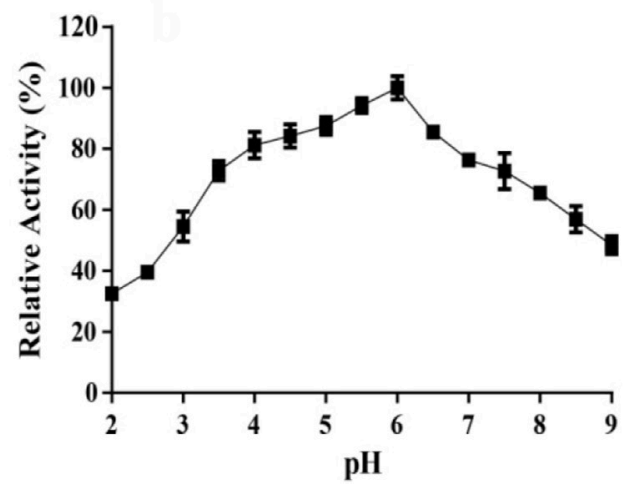

FIGURE 5 | Effects of pH on the activity (A) and stability (B) of TanA. (A) The optimal pH of TanA was investigated in the pH range of 2-11 with 100 mM buffers (glycine- $\mathrm{NaOH}, \mathrm{pH}$ 8.5-11.0; $\mathrm{Na}_{2} \mathrm{HPO}_{4}$-citric acid, $\mathrm{pH}$ 2.0-8.0) by taking the activity at the optimum $\mathrm{pH}$ as $100 \%$. (B) The pH stability of TanA was surveyed after incubation for $12 \mathrm{~h}$ at $40^{\circ} \mathrm{C}$ in the $\mathrm{pH}$ range of $2-11$ with the buffers described above; the highest residual activity was set as $100 \%$. The indifferent data ranging from $\mathrm{pH} 9$ to 11 were neglected and not shown in both figures $(\mathbf{A})$ and $(\mathbf{B})$.

$K_{\mathrm{m}}$ values of TanA against these substrates are listed in Table 2. TanA showed multifunctional property with high specific activities and affinities toward all the gallic acid esters utilized. Although the synthetic substrate PG demonstrated the highest specific activity of TanA at $941.4 \mathrm{U} / \mathrm{mg}$, it still exhibited considerable catalysis ability against other substrates, especially toward some natural substrates, such as GCG, ECG, and EGCG (Table 2). For further testing of the abilities of TanA to degrade diverse gallic acid esters, the HPLC analysis of degradation products is displayed in Supplementary Figure S3. After catalytic decomposition by Tan $\mathrm{A}$ at $55^{\circ} \mathrm{C}$ for $2 \mathrm{~h}$, no gallic acid ester could be detected in the reaction solutions, and all kinds of substrates were almost thoroughly transformed (Supplementary Figure S3). In the chromatograms of the transformation products after tannase TanA treatment against MG (Supplementary Figure S3A), PG (Supplementary Figure S3B), and TA (Supplementary Figure S3C), only the peaks of gallic acid could be revealed due to other degraded products (methanol for MG, propanol for PG, and glucose for TA) with no ultraviolet absorption. Significantly, the ester catechins that are ubiquitous in green tea, including GCG (Supplementary Figure S3D), ECG (Supplementary Figure S3E), and EGCG (Supplementary Figure S3F), were converted successfully to gallocatechin (GC), epicatechin (EC), and epigallocatechin (EGC), respectively, which belong to the non-eater catechins.

\section{DISCUSSION}

The previous study of the genes related to tannin degradation in A. melanogenum T9 has suggested that there existed several tannases (including TanA, TanB, and TanC) with different molecular masses, amino acid sequences, gene expression patterns, and diverse properties, which are together 


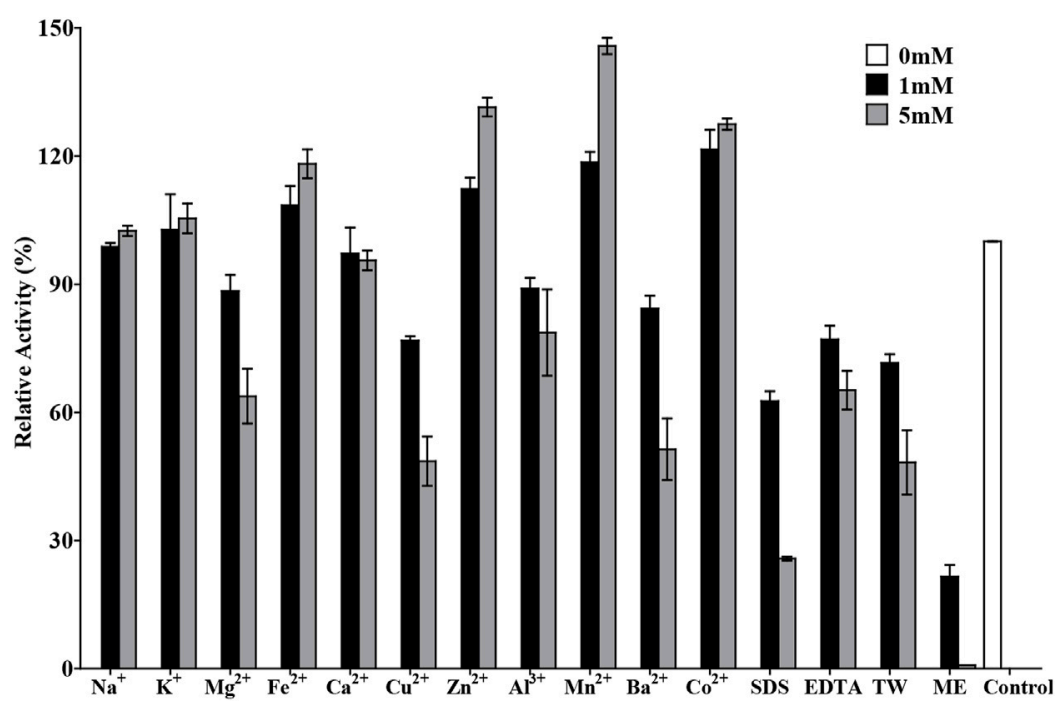

FIGURE 6 | Effects of chemicals and metal ions on the activity of TanA. The activity assayed without any chemical compound or metal ion was taken as control. SDS, sodium dodecyl sulfate; EDTA, ethylene diamine tetraacetic acid; TW, Tween 80; ME, mercaptoethanol.

TABLE 2 | Comparison of substrate specificities for TanA.

\begin{tabular}{lcc} 
Substrate & Specific activity $(\mathbf{U} / \mathbf{m g})$ & $\boldsymbol{K}_{\mathbf{m}}(\mathbf{m} \mathbf{M})$ \\
\hline TA & 723.8 & 2.15 \\
GCG & 703.1 & 1.86 \\
ECG & 756.2 & 2.13 \\
PG & 941.4 & 1.71 \\
MG & 506.8 & 2.81 \\
EGCG & 623.2 & 2.52
\end{tabular}

TA, tannic acid; GCG, gallocatechin gallate; ECG, epicatechin gallate; PG, propyl gallate; $M G$, methyl gallate; EGCG, epigallocatechin gallate.

responsible for the catalyzed hydrolysis of tannins in the strain T9 (Zhang et al., 2019). The tannases from different categories are often produced in the same fungal species due to species-specific accumulative effects and the impacts of exterior circumstances. Tannase subtypes are distinguished depending on their physical and chemical properties, such as molecular size, family, presence or absence of a signal peptide, and more, which guarantee that microorganisms are able to degrade both intra- and extracellular tannins under various physiological conditions, namely, temperature, $\mathrm{pH}$, ion strength, etc. Therefore, it is of great significance to figure out the metabolic mechanism of tannins in A. melanogenum in future work.

TanA exhibited a typical conserved domain, Gly-X-Ser-X-Gly, almost present in all tannase sequences of fungi and bacteria (boxed in red in Figure 1). This unique sequence of serine hydrolase effectively binds to the tannic substrates, thereby promoting catalytic activity (Zhang et al., 2019). Moreover, a mutation analysis explained that the disulfide bond between Cys202 and Cys458 played a vital part in regulating the activity of AoFaeB (Suzuki et al., 2014). Interestingly, TanA also showed several cysteine residues probably involved in disulfide bond formation at similar sites as that of AoFaeB.
For the sake of surveying this, the homology model of TanA was built. Meanwhile, four disulfide bonds with a 3D structure have been predicted (Supplementary Figure S2), including Cys161-Cys415, Cys230-Cys247, Cys256-Cys265, and Cys487-Cys508, among which the first disulfide bond was similar to the one in AoFaeB as described above, indicating that the disruption of disulfide bonds when subjected to ME affected TanA activity and stability to a great extent (Figure 6).

It is shown in Figure $\mathbf{4 A}$ that Tan A displayed an optimal response at $60^{\circ} \mathrm{C}$, with over $80 \%$ relative activity from 40 to $70^{\circ} \mathrm{C}$, illustrating its thermophilic property and making it suitable for most of the hydrolysis processes assisted by tannases which were carried out at increased temperatures (Abdulhameed et al., 2005). However, a lot of studies demonstrated that many tannases from bacteria (Jana et al., 2014), mold (Iibuchi et al., 1968; Lekha and Lonsane, 1997; Madzak, 2015; Sivashanmugam and Jayaraman, 2011), and even yeast (Wang et al., 2020) showed optimal activity at approximately $40^{\circ} \mathrm{C}$. Considering thermal stability, as exhibited in the diagram of Figure $\mathbf{4 B}$, TanA depicted superior heat resistance property at a temperature below $55^{\circ} \mathrm{C}$, maintaining up to $61.3 \%$ activity even after $12 \mathrm{~h}$ of incubation. Studies showed thermostability in the range of $30-50^{\circ} \mathrm{C}$ for most fungal tannases (Table 3). TanA possessed better temperature stability compared to other tannases from fungi. Although the tannase from $P$. notatum NCIM923 (Gayen and Ghosh, 2013) and the tannase from K. marxianus NRRL Y-8281 (Mahmoud et al., 2018) could maintain their stability up to 60 and $70^{\circ} \mathrm{C}$, respectively, according to their researches (Table 3), their thermostability at high temperatures was, in fact, worse than TanA. The purified tannase from $P$. notatum retained $60 \%$ of its thermostability at $60^{\circ} \mathrm{C}$ within only $1 \mathrm{~h}$ (Gayen and Ghosh, 2013). Meanwhile, the tannase from $K$. marxianus could also remain stable at $70^{\circ} \mathrm{C}$ for only $60 \mathrm{~min}$ (Mahmoud et al., 2018). Also belonging to thermophilic tannases, the extracellular tannase derived from 
TABLE 3 | Biochemical properties of TanA and other reported tannases.

\begin{tabular}{|c|c|c|c|c|c|}
\hline Source & $\begin{array}{l}\text { Specific activity } \\
(\mathrm{U} / \mathrm{mg})\end{array}$ & $\begin{array}{l}\text { Optimal pH/temperature } \\
\left({ }^{\circ} \mathrm{C}\right)\end{array}$ & $\begin{array}{l}\text { Temperature-stable range } \\
\left({ }^{\circ} \mathrm{C}\right)\end{array}$ & pH-stable range & References \\
\hline Penicillium notatum & 22.48 & $5.0 / 40$ & $30-60$ & $3.0-8.0$ & Gayen and Ghosh (2013) \\
\hline Emericella nidulans & 1.91 & $5.0 / 45$ & $22-50$ & $4.0-5.0$ & Goncalves et al. (2011) \\
\hline Aspergillus phoenicis & 10.0 & $6.0 / 60$ & $40-50$ & $2.5-7.0$ & Riul et al. (2013) \\
\hline A. niger & N.D. & $6.0 / 80$ & $30-60$ & $3.0-8.0$ & Shao et al. (2020) \\
\hline Sporidiobolus ruineniae & 16.232 & $7.0 / 40$ & $20-50$ & $5.0-9.0$ & Kanpiengjai et al. (2020) \\
\hline Kluyveromyces marxianus & $1,026.12$ & $4.5,8.5 / 35$ & $30-70$ & $4.0-6.0$ & Mahmoud et al. (2018) \\
\hline Streptomyces sviceus & 114.0 & $8.0 / 50$ & $63-69$ & $6.5-8.0$ & Wu et al. (2015) \\
\hline Lactobacillus plantarum & 84.34 & $8.0 / 40$ & N.D. & N.D. & Iwamoto et al. (2008) \\
\hline A. melanogenum & 941.4 & $6.0 / 60$ & $20-55$ & $3.5-7.5$ & This study \\
\hline
\end{tabular}

A. phoenicis, whose optimal temperature was the same as TanA, was stable for $1 \mathrm{~h}$ at $40-50^{\circ} \mathrm{C}$, with a half-life of only $20 \mathrm{~min}$ at $60^{\circ} \mathrm{C}$ (Riul et al., 2013). In industrial production such as green tea deep processing, the degradation of tannins often takes a longer time at a relatively high temperature, and the tannase TanA retained most of its activity within at least $12 \mathrm{~h}$ under $55^{\circ} \mathrm{C}$. This concludes that TanA is a thermophilic tannase with sturdy thermostability, which quite better satisfy the industrial application. Contrastingly, the optimal temperature for the tannase rAntan1, with strong thermal stability from $A$. niger FJ0118, was $80^{\circ} \mathrm{C}$. It could persist to be stable at $60^{\circ} \mathrm{C}$ for about $5.4 \mathrm{~h}$ (Shao et al., 2020). Although owning favorable activity and stability at high temperature, rAntan 1 expressed by Pichia pastoris cannot reach food grade because of the use of methanol when inducing tannase expression. The thermophilic and thermostable properties of TanA and rAntan 1 may be partially attributed to glycosylated formation during expression in yeast (Zhou et al., 2020), corresponding to the predicted $\mathrm{N}$-glycosylation recognition sites (Figure 1). Yang et al. (2007) indicated that the tea steeped in water at $70^{\circ} \mathrm{C}$ contained the highest contents of active components. Thus, the thermophilic and thermostable tannases, such as TanA and rAntan1, are rare in nature but necessary in industrial production. Some pieces of literature have reviewed the wide application of thermostable tannases in tea extracts to decrease the formation of tea cream (on account of the presence of tannins), thereby improving the taste of tea beverages (Chávez-González et al., 2012). In addition, thermo-tolerant tannases were also applied in the production of instant green tea powder to enhance the color and taste (Ozturk et al., 2016; Cao et al., 2019). In the process of catalytic degradation of tannins, thermostable tannases can enhance high-temperature extraction efficiency, simplify the production process, reduce the costs, and improve the product quality, which are reasons for the application of TanA in industrial production, such as in green tea deep processing.

TanA maintained over $60 \%$ activity within the broad $\mathrm{pH}$ scope of 3.0-7.0, with maximum activity at pH 6.0 (Figure 5A). For $\mathrm{pH}$ stability, TanA still retained over $70 \%$ of its activity after incubation for $12 \mathrm{~h}$ within the wide $\mathrm{pH}$ from 3.5 to 7.5 , indicating advisable stability under acidic to weakly alkaline conditions (Figure 5B). It was consistent with the reported fungal tannases-for instance, the tannase derived from A. niger possessed an optimal $\mathrm{pH}$ of 6.0 and excellent stability at $\mathrm{pH}$ 3.0-8.0 (Shao et al., 2020). The tannase from A. oryzae had an optimum $\mathrm{pH}$ of 5.5 along with stability between $\mathrm{pH} 4.5$ and 7.5 and over $80 \%$ activity retention (Abdel-Naby et al., 2016). On the other hand, the tannase from E. nidulans ( $\mathrm{pH} 4.0-5.0$ ) and the tannase from Aspergillus thorny ( $\mathrm{pH}$ 5.0-6.0) displayed limited pH stability (Goncalves et al., 2011; Tanash et al., 2011). Therefore, remarkable stability at high temperatures and a wide $\mathrm{pH}$ range become integral properties of TanA, which reduce the risk of pollution during the fermentation process and thereby establish the foundation for its industrial application in tannin biodegradation and gallic acid preparation.

TanA has been incubated with several gallic acid esters to study the substrate specificity. As shown in Table 2, TanA revealed outstanding specific activities to decompose various substrates, illustrating its multifunctional property. The activity of TanA on synthetic substrate PG was significantly superior, whereas the specific activities on some natural substrates (e.g., 703.1 U/mg against GCG, 756.2 U/mg against ECG, and 623.2 $\mathrm{U} / \mathrm{mg}$ against EGCG) were also quite considerable (Table 2). Many reported tannases exhibited quite lower activities toward some ester catechins that naturally exist in green tea, such as ECG and EGCG (Bhoite et al., 2013; Ramírez-Coronel et al., 2003; Sharma et al., 2008). Although Mahmoud et al. (2018) stated a high activity toward TA $(1,026.12 \mathrm{U} / \mathrm{mg})$, the tannase from $K$. marxianus NRRL Y-8281 was most active on TA (100\%), followed by $\mathrm{MG}$ (74.3\%) and PG (64.5\%) and expressing minimum enzymatic activity against EGCG (only 10.5\%). Obviously, these enzymes cannot satisfy the application in industrial processing when against natural ester catechins. In addition, the HPLC analysis results of transformation products by TanA further confirmed the almost complete degradation of varying kinds of tannins (especially the natural ester catechins in many plants) at a high temperature within $2 \mathrm{~h}$ (Supplementary Figure S3). Previous research reported that immobilized tannase could carry out $98 \%$ conversion of TA within $6 \mathrm{~h}$ (Mahendran et al., 2006). However, tannase from Bacillus sphaericus converted 90.8\% TA after as long as $24 \mathrm{~h}$ (Raghuwanshi et al., 2011). By contrast, the higher efficiency of TanA, along with high bioconversion, undoubtedly benefited from its favorable activities toward different gallic acid esters. Moreover, plants 
in nature, such as oilseed rape and many fruits, usually contain various hydrolyzable tannins (GTs and ETs) (Lorusso et al., 1996). Thus, the multifunction property gives the tannase TanA the capability to decompose diverse gallic acid esters, which is fully conducive when against different kinds of tannins in the plants. The prime cause of the bitter taste of tea is the presence of ester catechins (mainly EGCG and ECG) (Shao et al., 2020). The reported tannase rAntan 1 displayed similar substrate specificity as TanA. Enzymatic extraction reduced the proportion of easter catechins in tea polyphenols, weakened the bitter taste, and improved the overall taste of the tea beverage (Shao et al., 2020). Similarly, the TanA-mediated extraction processing can also effectively transform the ester catechins to the non-ester ones (such as EGC and EC) in green tea, thereby improving the taste of tea drinks. Besides this, the green tea catechins possess many biological activities, such as antiviral, antibacterial, antitumor, and antioxidant properties, which are closely related to the presence of multiple hydroxyl groups and galloyl groups in their structures (Xu et al., 2021).

Tannins are ubiquitously present in higher plants, and biodegradation by tannases is essential in industrial processing, such as in feed additives (Lorusso et al., 1996), myrobalan juice (Srivastava and Kar, 2009), and green tea (Cao et al., 2019; Shao et al., 2020). In addition, many active substances, such as catechins and gallic acid, employ tannases for their respective extraction (Chhokar et al., 2013). Rapeseed meal processing is a worthy example illustrating the application of tannases. The agro-industrial chain of canola oil production generates a large mass of rapeseed meals but faces challenges during recycling owing to the high content of tannins, resulting in huge wastes (Lorusso et al., 1996; Shim et al., 2003). Fortunately, the efficient decomposition of tannins through enzymatic catalysis aids in the production of rapeseed meal feed with added value (Lorusso et al., 1996). In other words, tannases play significant roles in various fields, such as food, brewing, pharmacy, and feed. However, tannases of food grade with advisable properties are still rather rare but necessary. In this research, a novel thermophilic tannase, TanA, with favorable thermostability was demonstrated, with detailed characterizations suggesting a potent candidate for the food and agricultural industries.

\section{REFERENCES}

Abdel-Naby, M. A., El-Tanash, A. B., Sherief, A. D. A., and Sherief, A. (2016). Structural Characterization, Catalytic, Kinetic and Thermodynamic Properties of Aspergillus Oryzae Tannase. Int. J. Biol. Macromolecules 92, 803-811. doi:10. 1016/j.ijbiomac.2016.06.098

Abdulhameed, S., Kiran, G., and Pandey, A. (2005). Purification and Characterization of Tannin Acyl Hydrolase from Aspergillus Niger ATCC 16620. Food Technol. Biotech. 43, 133-138. doi:10.1016/j.fm.2004.02.005

Aguilar, C. N., and Gutierrez-Sanchez, G. (2001). Review: Sources, Properties, Applications and Potential Uses of Tannin Acyl Hydrolase. Food Sci. Technol. Int. 7, 373-382. doi:10.1106/69m3-b30k-cf7q-ri5g

Banerjee, A., Jana, A., Pati, B. R., Mondal, K. C., and Das Mohapatra, P. K. (2012). Characterization of Tannase Protein Sequences of Bacteria and Fungi: An In Silico Study. Protein J. 31, 306-327. doi:10.1007/s10930-0129405-x

\section{DATA AVAILABILITY STATEMENT}

The datasets presented in this study can be found in online repositories. The names of the repository/repositories and accession number(s) can be found below: https:/www.ncbi. nlm.nih.gov/genbank/, QEP28943.1.

\section{AUTHOR CONTRIBUTIONS}

LL contributed to methodology, formal analysis, validation, writing of the original draft, and in review and editing. JG contributed to conceptualization, investigation, and manuscript review and revision. X-FZ contributed to data curation, and manuscript review and revision. ZL contributed to software. W-QS contributed to methodology, resources, supervision, and project administration. $\mathrm{H}-\mathrm{XZ}$ contributed to methodology, resources, review and editing, supervision, and project administration.

\section{FUNDING}

This research was funded by the Shandong Provincial Natural Science Foundation, China (ZR2016BQ42, ZR2020MC003, and ZR2017BC029) and the National Key R and D Program of China (2019YFD0901902).

\section{ACKNOWLEDGMENTS}

The authors would like to thank all the reviewers and editors who participated in the review and MJEditor (www.mjeditor.com) for its linguistic assistance during the preparation of this manuscript.

\section{SUPPLEMENTARY MATERIAL}

The supplementary material for this article can be found online at: https:/www.frontiersin.org/articles/10.3389/fbioe.2021.769816/ full\#supplementary-material

Becker, K., and Makkar, H. P. S. (1999). Effects of Dietary Tannic Acid and Quebracho Tannin on Growth Performance and Metabolic Rates of Common Carp (Cyprinus carpio L.). Aquaculture 175, 327-335. doi:10.1016/S00448486(99)00106-4

Beniwal, V., Chhokar, V., Singh, N., and Sharma, J. (2010). Optimization of Process Parameters for the Production of Tannase and Gallic Acid by Enterobacter Cloacae MTTC 9125. Biocatal. Agric. Biotechnol. 6, 389-397. doi:10.1007/ s13297-017-2490-2

Beniwal, V., Kumar, A., Goel, G., and Chhokar, V. (2013). A Novel Low Molecular Weight Acido-Thermophilic Tannase from Enterobacter Cloacae MTCC 9125. Biocatal. Agric. Biotechnol. 2, 132-137. doi:10.1016/j.bcab.2013.03.002

Bhoite, R. N., Navya, P., and Murthy, P. S. (2013). Purification and Characterisation of a Coffee Pulp Tannase Produced by Penicillium Verrucosum. J. Food Sci. Eng. 3, 323-331. doi:10.1016/j.fbp.2013.10.007

Cao, Q.-Q., Zou, C., Zhang, Y.-H., Du, Q.-Z., Yin, J.-F., Shi, J., et al. (2019). Improving the Taste of Autumn Green tea with Tannase. Food Chem. 277, 432-437. doi:10.1016/j.foodchem.2018.10.146 
Chávez-González, M., Rodríguez-Durán, L. V., Balagurusamy, N., Prado-Barragán, A., Rodríguez, R., Contreras, J. C., et al. (2012). Biotechnological Advances and Challenges of Tannase: An Overview. Food Bioproc. Technol 5, 445-459. doi:10. 1007/s11947-011-0608-5

Chhokar, V., Sharma, J., Kumar, A., and Beniwal, V. (2013). Recent Advances in Industrial Application of Tannases: A Review. Recent Pat. Biotechnol. 7, 228-233. doi:10.2174/18722083113076660013

Chung, K.-T., Wong, T. Y., Wei, C.-I., Huang, Y.-W., and Lin, Y. (1998). Tannins and Human Health: A Review. Crit. Rev. Food Sci. Nutr. 38, 421-464. doi:10. 1080/10408699891274273

Ebrahimzadeh, S. K., Navidshad, B., Farhoomand, P., and Mirzaei Aghjehgheshlagh, F. (2018). Effects of Exogenous Tannase Enzyme on Growth Performance, Antioxidant Status, Immune Response, Gut Morphology and Intestinal Microflora of Chicks Fed Grape Pomace. S. Afr. J. Anim. Sci. 48, 2-9. doi:10.4314/sajas.v48i1.2

Gayen, S., and Ghosh, U. (2013). Purification and Characterization of Tannin Acyl Hydrolase Produced by Mixed Solid State Fermentation of Wheat Bran and Marigold Flower byPenicillium notatumNCIM 923. Biomed. Res. Int. 2013, 1-6. doi:10.1155/2013/596380

Ghosh, K., and Mandal, S. (2015). Nutritional Evaluation of Groundnut Oil Cake in Formulated Diets for Rohu, Labeo Rohita (Hamilton) Fingerlings after Solid State Fermentation with a Tannase Producing Yeast, Pichia Kudriavzevii (GU939629) Isolated from Fish Gut. Aquacult. Rep. 2, 82-90. doi:10.1016/j. aqrep.2015.08.006

Gonçalves, H. B., Riul, A. J., Terenzi, H. F., Jorge, J. A., and Guimarães, L. H. S. (2011). Extracellular Tannase from Emericella Nidulans Showing Hypertolerance to Temperature and Organic Solvents. J. Mol. Catal. B: Enzymatic 71, 29-35. doi:10.1016/j.molcatb.2011.03.005

Govindarajan, R. K., Revathi, S., Rameshkumar, N., Krishnan, M., and Kayalvizhi, N. (2016). Microbial Tannase: Current Perspectives and Biotechnological Advances. Biocatal. Agric. Biotechnol. 6, 168-175. doi:10.1016/j.bcab.2016. 03.011

Hong, Y.-H., Jung, E. Y., Park, Y., Shin, K.-S., Kim, T. Y., Yu, K.-W., et al. (2013). Enzymatic Improvement in the Polyphenol Extractability and Antioxidant Activity of Green Tea Extracts. Biosci. Biotechnol. Biochem. 77, 22-29. doi:10. 1271/bbb.120373

Iibuchi, S., Minoda, Y., and Yamada, K. (1968). Studies on Tannin Acyl Hydrolase of Microorganisms. Agric. Biol. Chem. 32, 803-809. doi:10.1271/bbb1961. 32.803

Iwamoto, K., Tsuruta, H., Nishitaini, Y., and Osawa, R. (2008). Identification and Cloning of a Gene Encoding Tannase (Tannin Acylhydrolase) from Lactobacillus Plantarum ATCC 14917T. Syst. Appl. Microbiol. 31, 269-277. doi:10.1016/j.syapm.2008.05.004

Jana, A., Maity, C., Halder, S. K., Das, A., Pati, B. R., Mondal, K. C., et al. (2013). Structural Characterization of Thermostable, Solvent Tolerant, Cytosafe Tannase from Bacillus Subtilis PAB2. Biochem. Eng. J. 77, 161-170. doi:10. 1016/j.bej.2013.06.002

Jana, A., Halder, S. K., Banerjee, A., Paul, T., Pati, B. R., Mondal, K. C., et al. (2014). Biosynthesis, Structural Architecture and Biotechnological Potential of Bacterial Tannase: A Molecular Advancement. Bioresour. Technol. 157, 327-340. doi:10.1016/j.biortech.2014.02.017

Kanpiengjai, A., Unban, K., Nguyen, T.-H., Haltrich, D., and Khanongnuch, C. (2019). Expression and Biochemical Characterization of a New Alkaline Tannase from Lactobacillus Pentosus. Protein Expr. Purif. 157, 36-41. doi:10.1016/j.pep.2019.01.005

Kanpiengjai, A., Khanongnuch, C., Lumyong, S., Haltrich, D., Nguyen, T.-H., and Kittibunchakul, S. (2020). Co-Production of Gallic Acid and a Novel CellAssociated Tannase by a Pigment-Producing Yeast, Sporidiobolus Ruineniae A45.2. Microb. Cel Fact. 19, 95-99. doi:10.1186/s12934-020-01353-w

Lekha, P. K., and Lonsane, B. K. (1997). Production and Application of Tannin Acyl Hydrolase: State of the Art. Adv. Appl. Microbiol. 44, 215-260. doi:10. 1016/S0065-2164(08)70463-5

Lineweaver, H., and Burk, D. (1934). The Determination of Enzyme Dissociation Constants. J. Am. Chem. Soc. 56, 658-666. doi:10.1021/ja01318a036

Lorusso, L., Lacki, K., and Duvnjak, Z. (1996). Decrease of Tannin Content in Canola Meal by an Enzyme Preparation from Trametes Versicolor. Biotechnol. Lett. 18, 309-314. doi:10.1007/BF00142950
Madzak, C. (2015). Yarrowia Lipolytica: Recent Achievements in Heterologous Protein Expression and Pathway Engineering. Appl. Microbiol. Biotechnol. 99, 4559-4577. doi:10.1007/s00253-015-6624-z

Mahendran, B., Raman, N., and Kim, D.-J. (2006). Purification and Characterization of Tannase from Paecilomyces Variotii: Hydrolysis of Tannic Acid Using Immobilized Tannase. Appl. Microbiol. Biotechnol. 70, 444-450. doi:10.1007/s00253-005-0082-y

Mahmoud, A. E., Fathy, S. A., Rashad, M. M., Ezz, M. K., and Mohammed, A. T. (2018). Purification and Characterization of a Novel Tannase Produced by Kluyveromyces Marxianus Using Olive Pomace as Solid Support, and its Promising Role in Gallic Acid Production. Int. J. Biol. Macromolecules 107, 2342-2350. doi:10.1016/j.ijbiomac.2017.10.117

Min, B. R., Attwood, G. T., Mcnabb, W. C., Molan, A. L., and Barry, T. N. (2005). The Effect of Condensed Tannins from Lotus Corniculatus on the Proteolytic Activities and Growth of Rumen Bacteria. Anim. Feed Sci. Technol. 121, 45-58. doi:10.1016/j.anifeedsci.2005.02.007

Ni, H., Chen, F., Jiang, Z. D., Cai, M. Y., Yang, Y. F., Xiao, A. F., et al. (2015). Biotransformation of tea Catechins Using Aspergillus Niger Tannase Prepared by Solid State Fermentation on tea Byproduct. LWT - Food Sci. Technol. 60, 1206-1213. doi:10.1016/j.lwt.2014.09.010

Ozturk, B., Seyhan, F., Ozdemir, I. S., Karadeniz, B., Bahar, B., Ertas, E., et al. (2016). Change of Enzyme Activity and Quality during the Processing of Turkish Green tea. LWT - Food Sci. Technol. 65, 318-324. doi:10.1016/j.lwt. 2015.07.068

Pan, J., Wang, N.-N., Yin, X.-J., Liang, X.-L., and Wang, Z.-P. (2020). Characterization of a Robust and $\mathrm{pH}$-Stable Tannase from MangroveDerived Yeast Rhodosporidium Diobovatum Q95. Mar. Drugs 18, 546-552. doi:10.3390/md18110546

Raghuwanshi, S., Dutt, K., Gupta, P., Misra, S., and Saxena, R. K. (2011). Bacillus Sphaericus: The Highest Bacterial Tannase Producer with Potential for Gallic Acid Synthesis. J. Biosci. Bioeng. 111, 635-640. doi:10.1016/j.jbiosc.2011.02.008

Ramírez-Coronel, M. A., Viniegra-González, G., Darvill, A., and Augur, C. (2003). A Novel Tannase from Aspergillus niger with $\beta$-Glucosidase Activity. Microbiology 149, 2941-2946. doi:10.1099/mic.0.26346-0

Riul, A. J., Gonçalves, H. B., Jorge, J. A., and Guimarães, L. H. S. (2013). Characterization of a Glucose- and Solvent-Tolerant Extracellular Tannase from Aspergillus Phoenicis. J. Mol. Catal. B: Enzymatic 85-86, 126-133. doi:10. 1016/j.molcatb.2012.09.001

Rivas, B. D. L., Rodríguez, H., Anguita, J., and Muñoz, R. (2019). Bacterial Tannases: Classification and Biochemical Properties. Appl. Microbiol. Biotechnol. 103, 603-623. doi:10.1007/s00253-018-9519-y

Şengil, İ. A., and Özacar, M. (2009). Competitive Biosorption of $\mathrm{Pb} 2+, \mathrm{Cu} 2+$ and Zn2+ Ions from Aqueous Solutions onto Valonia Tannin Resin. J. Hazard. Mater. 166, 1488-1494. doi:10.1016/j.jhazmat.2008.12.071

Shao, Y., Zhang, Y.-H., Zhang, F., Yang, Q.-M., Weng, H.-F., Xiao, Q., et al. (2020). Thermostable Tannase from Aspergillus Niger and its Application in the Enzymatic Extraction of Green tea. Molecules 25, 952-969. doi:10.3390/ molecules 25040952

Sharma, S., Bhat, T. K., and Dawra, R. K. (2000). A Spectrophotometric Method for Assay of Tannase Using Rhodanine. Anal. Biochem. 279, 85-89. doi:10.1006/ abio.1999.4405

Sharma, S., Agarwal, L., and Saxena, R. K. (2008). Purification, Immobilization and Characterization of Tannase from Penicillium Variable. Bioresour. Technol. 99, 2544-2551. doi:10.1016/j.biortech.2007.04.035

Shim, Y. H., Chae, B. J., and Lee, J. H. (2003). Effects of Phytase and Carbohydrases Supplementation to Diet with a Partial Replacement of Soybean Meal with Rapeseed Meal and Cottonseed Meal on Growth Performance and Nutrient Digestibility of Growing Pigs. Asian Australas. J. Anim. Sci. 16, 1339-1347. doi:10.5713/ajas.2003.1339

Sivashanmugam, K., and Jayaraman, G. (2011). Production and Partial Purification of Extracellular Tannase by Klebsiella Pneumoniae MTCC 7162 Isolated from Tannery Effluent. Afr. J. Biotechnol. 10, 1364-1374. doi:10.1186/1471-218011-37

Srivastava, A., and Kar, R. (2009). Characterization and Application of Tannase Produced by Aspergillus Niger ITCC 6514.07 on Pomegranate Rind. Braz. J. Microbiol. 40, 782-789. doi:10.1590/S1517-83822009000400008 
Suzuki, K., Hori, A., Kawamoto, K., Thangudu, R. R., Ishida, T., Igarashi, K., et al. (2014). Crystal Structure of a Feruloyl Esterase Belonging to the Tannase Family: A Disulfide Bond Near a Catalytic Triad. Proteins 82, 2857-2867. doi:10.1002/prot.24649

Tamura, K., Stecher, G., Peterson, D., Filipski, A., and Kumar, S. (2013). MEGA6: Molecular Evolutionary Genetics Analysis Version 6.0. Mol. Biol. Evol. 30, 2725-2729. doi:10.1093/molbev/mst197

Tanash, D. A., Sherief, A., and Nour, A. (2011). Catalytic Properties of Immobilized Tannase Produced from Aspergillus Aculeatus Compared with the Free Enzyme. Braz. J. Chem. Eng. 28, 381-391. doi:10.1590/S010466322011000300004

Wang, Z.-P., Cao, M., Li, B., Ji, X.-F., Zhang, X.-Y., Zhang, Y.-Q., et al. (2020). Cloning, Secretory Expression and Characterization of a Unique pH-Stable and Cold-Adapted Alginate Lyase. Mar. Drugs 18, 189. doi:10.3390/ md18040189

Wang, Z.-P., Wang, P.-K., Ma, Y., Lin, J.-X., Wang, C.-L., Zhao, Y.-X., et al. (2022). Laminaria Japonica Hydrolysate Promotes Fucoxanthin Accumulation in Phaeodactylum Tricornutum. Bioresour. Technol. 344, 126117. doi:10.1016/j. biortech.2021.126117

Wu, M., Wang, Q., Mckinstry, W. J., and Ren, B. (2015). Characterization of a Tannin Acyl Hydrolase from Streptomyces Sviceus with Substrate Preference for Digalloyl Ester Bonds. Appl. Microbiol. Biotechnol. 99, 2663-2672. doi:10.1007/ s00253-014-6085-9

Xu, Y.-Q., Gao, Y., and Granato, D. (2021). Effects of Epigallocatechin Gallate, Epigallocatechin and Epicatechin Gallate on the Chemical and Cell-Based Antioxidant Activity, Sensory Properties, and Cytotoxicity of a CatechinFree Model Beverage. Food Chem. 339, 128060. doi:10.1016/j.foodchem. 2020.128060

Yang, D.-J., Hwang, L. S., and Lin, J.-T. (2007). Effects of Different Steeping Methods and Storage on Caffeine, Catechins and Gallic Acid in Bag tea Infusions. J. Chromatogr. A 1156, 312-320. doi:10.1016/j.chroma.2006.11.088

Zhang, S., Gao, X., He, L., Qiu, Y., Zhu, H., and Cao, Y. (2015). Novel Trends for Use of Microbial Tannases. Prep. Biochem. Biotechnol. 45, 221-232. doi:10. 1080/10826068.2014.907182
Zhang, P., Wang, Z.-P., Sheng, J., Zheng, Y., Ji, X.-F., Zhou, H.-X., et al. (2018) High and Efficient Isomaltulose Production Using an Engineered Yarrowia Lipolytica Strain. Bioresour. Technol. 265, 577-580. doi:10.1016/j.biortech.2018. 06.081

Zhang, L.-L., Li, J., Wang, Y.-L., Liu, S., Wang, Z.-P., and Yu, X.-J. (2019). Integrated Approaches to Reveal Genes Crucial for Tannin Degradation in Aureobasidium Melanogenum T9. Biomolecules 9, 439-445. doi:10.3390/ biom9090439

Zhang, L.-L., Jiang, X.-H., Xiao, X.-F., Zhang, W.-X., Shi, Y.-Q., Wang, Z.-P., et al. (2021). Expression and Characterization of a Novel Cold-Adapted Chitosanase from marine Renibacterium Sp. Suitable for Chitooligosaccharides Preparation. Mar. Drugs 19, 596. doi:10.3390/md19110596

Zhou, H.-X., Xu, S.-S., Yin, X.-J., Wang, F.-L., and Li, Y. (2020). Characterization of a New Bifunctional and Cold-Adapted Polysaccharide Lyase (PL) Family 7 Alginate Lyase from Flavobacterium Sp. Mar. Drugs 18, 388. doi:10.3390/ md18080388

Conflict of Interest: The authors declare that the research was conducted in the absence of any commercial or financial relationships that could be construed as a potential conflict of interest.

Publisher's Note: All claims expressed in this article are solely those of the authors and do not necessarily represent those of their affiliated organizations or those of the publisher, the editors, and the reviewers. Any product that may be evaluated in this article or claim that may be made by its manufacturer is not guaranteed or endorsed by the publisher.

Copyright (c) 2022 Liu, Guo, Zhou, Li, Zhou and Song. This is an open-access article distributed under the terms of the Creative Commons Attribution License (CC BY). The use, distribution or reproduction in other forums is permitted, provided the original author(s) and the copyright owner(s) are credited and that the original publication in this journal is cited, in accordance with accepted academic practice. No use, distribution or reproduction is permitted which does not comply with these terms. 